transitions can be observed to infer all four $W_{i}$ 's, the relationships given will be of use in data analysis.

\footnotetext{
* This investigation was supported in part by the Air Force Office of Scientific Research (ARDC) under contract.

${ }^{1}$ B. Bleaney and K. W. H. Stevens, Repts. Progr. in Phys. 16, 108 (1951)

${ }_{2}^{2}$ R. Stahl-Brada and W. Low, Nuovo cimento Suppl. 15, 290 $(1960)$.

${ }^{3}$ E. O. Schulz-DuBois, Bell System Tech. J. 33, 271 (1959).

${ }^{4}$ W. S. Chang and A. E. Siegman, Stanford Electronics Laboratories, Stanford, California, Tech. Rept. No. 156-2.

B P. M. Parker and L. C. Brown, Am. J. Phys. 27, 509 (1959).
}

\section{Nuclear Magnetic Resonance Spectrum of the Triphenylcarbonium Ion*}

\author{
R. S. Berry, $\dagger$ R. Dehl, and W. R. Vaughan \\ Department of Chemistry, University of Michigan, \\ Ann Arbor, Michigan
}

(Received December 15, 1960)

$\mathbf{T}$ HE relative order of the chemical shifts in the ring spectrum of the triphenylcarbonium ion was estimated by Moodie, Connor, and Stewart ${ }^{1}$ and measured by us $^{2}$ from appropriately deuterated species. The chemical shifts which we originally reported are essentially correct: $\delta_{\text {para-ortho }}=0.525 \mathrm{ppm}$ and $\delta_{\text {para-meta }}=$ $0.355 \mathrm{ppm}$. However, the coupling constants were in error. We have reevaluated the latter by the method of total reproduction of the NMR spectrum, based on the complete solution of the secular equation for the system $\mathrm{AB}_{2} \mathrm{C}_{2}$. The calculations followed the general procedure used by Schneider, Bernstein, and Pople ${ }^{3}$ for pyridine, an $A B_{2} X_{2}$ spectrum. Our spin wave functions and Hamiltonian matrix elements were identical to those used in the pyridine case, except for the inclusion of a few additional off-diagonal matrix elements, made necessary by the removal of the $X$ approximation for $A B_{2} X_{2}$.

Table $I$ is a list of the additional nonvanishing matrix elements for $A B_{2} C_{2}$ in the spin wave notation of Schneider, Bernstein, and Pople. ${ }^{3}$

The diagonalization of the submatrices of the complete Hamiltonian and the computation of line intensities were performed on an IBM 704 computer for several sets of trial coupling constants, using the measured relative chemical shifts. Line intensities were calculated for each of the $\mathbf{1 1 0}$ transitions allowed by the selection rule $\Delta F_{z}= \pm 1$, where $F_{z}$ is the total $z$ component of angular momentum of a given spin wave function. In order to approximate the general appearance of an NMR spectrum, we drew a smooth curve through points which were evaluated by (1) plotting a Lorentz curve about each of the computed transition intensities, using a single value of $T_{2}$ estimated from the experimental linewidths and, (2), adding up the contributions from all 110 curves at small regular intervals over the span of the spectrum. The experimental $60-\mathrm{Mc}$ spectrum of triphenylcarbonium is shown in Fig. 1(a), together with the calculated spectrum [Fig. 1(b)] which was judged a best fit after several trial sets of parameters.

The value used for $T_{2}$ was $0.4 \mathrm{sec}$, and the chemical shifts in cps were as follows: $J_{o m}=8.2, J_{o m}=0.5$, $J_{m p}=8.0, J_{o p}=1.2, J_{m m^{\prime}}=1.7, J_{o o}=1.2$. The primes are used to denote a proton on the opposite side of the ring; $J_{o m^{\prime}}$ is very small and could be negative, but the signs of the other $J$ 's are all apparently positive, and correct within \pm 0.5 cps.

The relative chemical shifts of the ortho and meta protons are the reverse of those estimated by O'Reilly and Leftin, ${ }^{4}$ but their alternative assignments are in good agreement with ours. A complete spectral calculation identical to that of Fig. 1(b), except for the reversal of the meta and ortho shifts, yields quite a different spectrum [Fig. 1(c)] which does not agree well with the observed one. Using these authors' calculations for the

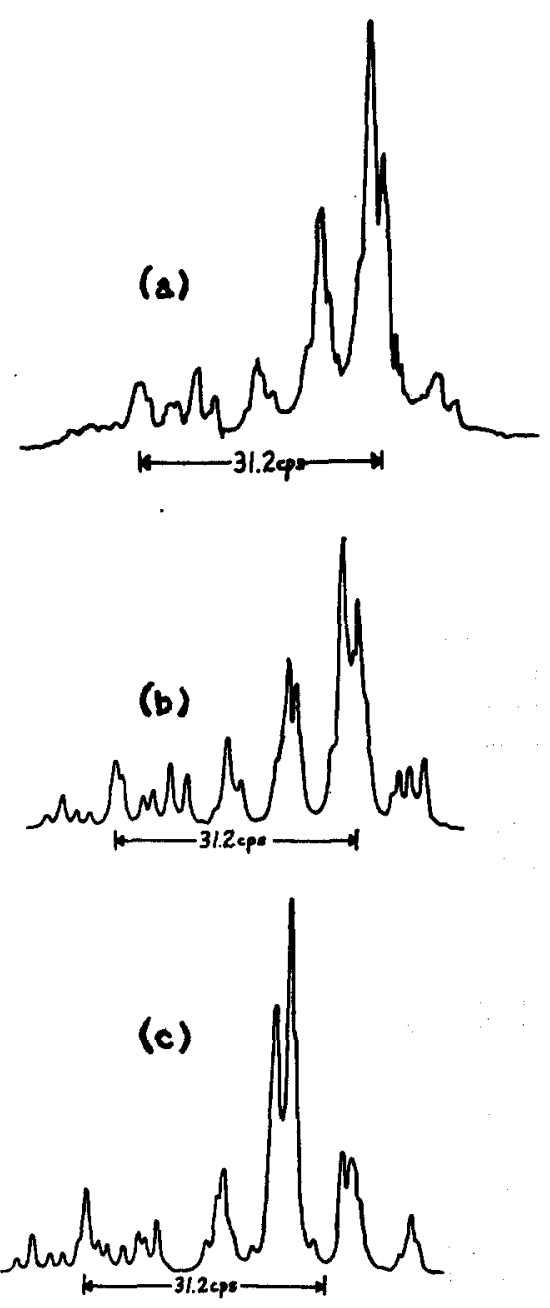

FIG. 1. (a) 60-Mc NMR spectrum of the triphenylcarbonium ion. (b) Calculated $60-\mathrm{Mc}$ spectrum. (c) Calculated 60-Mc spectrum with ortho and meta chemical shifts reversed. 
TABLE I. Off-diagonal matrix elements of the $A B_{2} C_{2}$ Hamiltonian not included in the $A B_{2} X_{2}$ approximation.

\begin{tabular}{|c|c|}
\hline Symmetric & Antisymmetric \\
\hline$\left(1 s_{3}|H| 2 s_{1}\right)=\left(4 s_{1}|H| 6 s_{\frac{1}{2}}\right)$ & $\left(1 a_{1}|H| 2 a_{3}\right)$ \\
\hline$=\left(1 s_{-\frac{1}{2}}: H \mid 3 s_{-3}\right)$ & $=\left(3 a_{\frac{1}{3}}|H| 4 a_{\frac{1}{2}}\right)$ \\
\hline$=\left(2 s_{-1}|H| 3 s_{-1}\right)=\frac{1}{2}\left(J_{u m n}+J_{o m^{\prime}}\right)$ & $=\left(1 a_{-\frac{1}{1}}|H| 2 a_{-\frac{1}{2}}\right)$ \\
\hline $\begin{array}{l}\left(1 s_{3}|H| 3 s_{3}\right)=\left(1 s_{\frac{1}{2}}|H| 4 s_{1}\right) \\
=\left(3 s_{-\frac{1}{3}}|H| 6 s_{-\frac{1}{3}}\right)\end{array}$ & $\begin{array}{l}=\left(1 a_{-1}|H| 2 a_{-i}\right) \\
=\frac{1}{2}\left(J_{o m}-J_{o m^{\prime}}\right)\end{array}$ \\
\hline$=\left(1 s_{-1}|H| 3 s_{-i}\right)=1 / \sqrt{2} J_{o p}$ & $\begin{array}{l}\left(1 a_{1}|I| 4 a_{1}\right) \\
=\left(2 a_{\frac{1}{2}}|H| 4 a_{1}\right)\end{array}$ \\
\hline$\left(1 s_{\frac{1}{2}}|H| 3 s_{\frac{1}{2}}\right)=\left(3 s_{\frac{1}{2}}|H| 5 s_{\frac{1}{2}}\right)$ & $=\left(1 a_{-1}|H| 3 a_{-\frac{1}{2}}\right)$ \\
\hline$=\left(2 s_{-1}|H| 4 s_{-1}\right)$ & $=\left(1 a_{-1}|H| 4 a_{-1}\right)$ \\
\hline$=\left(4 s_{-\frac{1}{2}}|I| 6 s_{-\frac{1}{2}}\right)=1 / \sqrt{2} J_{o m}$ & $=\frac{1}{2} J_{o p}$ \\
\hline \multicolumn{2}{|l|}{$\left(2 s_{2}^{i}|I| \sigma s_{\frac{1}{2}}\right)=\left(3 s_{\frac{1}{3}}|I| 6 s_{3}\right)$} \\
\hline \multicolumn{2}{|l|}{$=\left(1 s_{-1} \cdot I I \mid 4 s_{-1}\right)$} \\
\hline \multicolumn{2}{|l|}{$=\left(1 s_{-1}|H| 5 s_{-\frac{1}{3}}\right)=\frac{1}{2} J_{v p}$} \\
\hline \multicolumn{2}{|l|}{$\left(1 s_{\frac{1}{2}}|H| 2 s_{1}\right)=\left(2 s_{1}|H| 5 s_{\frac{1}{3}}\right)$} \\
\hline \multicolumn{2}{|l|}{$=\left\langle 2 s_{-\frac{1}{2}}|H| 5 s_{-1}\right\rangle$} \\
\hline$=\left(5 s_{-1}|H| 6 s_{-\frac{1}{3}}\right)=1 / \sqrt{2} J_{o m^{\prime}}$ & \\
\hline
\end{tabular}

ring-current contribution to the chemical shifts, we suggest that the discrepancy may be resolved by asuming the slightly larger value of $1.2 \mathrm{~A}$ for the effective van der Waals radius of hydrogen, which increases the average inclination angle of the benzene rings to about $30^{\circ}$ from the horizontal.

* Supported in part by a grant from the University of Michigan Cancer Research Institute.

$\dagger$ Present address: Sterling Chemistry Laboratory, Yale University, New Haven, Connecticut.

I R. B. Moodie, T. M. Connor, and Ross Stewart, Can. J. Chem. 37, 1402 (1959).

${ }^{2}$ R. Dehl, W. R. Vaughan, and R. S. Berry, J. Org. Chem. 24, 1616 (1959).

${ }^{3}$ W. G. Schneider, H. J. Bernstein, and J. A. Pople, Can. J. Chem. 35, 1487 (1957).

${ }^{4}$ D. E. O'Reilly and H. P. Leftin, J. Phys. Chem. 64, 1555 $(1960)$.

\section{Intrinsic Viscosity of Stiff Chains}

\author{
Robert Ullman and A. Muzyka
}

The Institute for Polymer Research, Department of Chemistry, and Department of Mathematics, Polytechnic Institute of Brooklyn, Brooklyn, New York

(Received November 21, 1960)

$\mathbf{T}$ HE intrinsic viscosity of a solution of partially coiled polymer molecules is being investigated by us using a procedure developed by Burgers ${ }^{1}$ and extended by Kirkwood and Riseman. ${ }^{2}$ The coiling of the polymer molecules is described by the chain statistics of Hermans and Ullman. ${ }^{3}$ In this treatment, two parameters are important, the length $n$ and a quantity $\lambda$ which is large when the chain is tightly coiled and is small when the chain becomes straight. Specifically, if $\theta$ is the angle a tangent to the chain makes with an arbitrary axis, $\lambda$ is one quarter of the average square change in $\theta$ per unit of chain (see p. 958 of the work cited in footnote 3 ).

The formal treatment is similar to that of Kirkwood and Riseman, the solution of the problem depending on an inhomogeneous integral equation of the Fredholm type. The result may be written

$$
\begin{array}{r}
{[\eta]=-\left(N / 100 M_{0 \eta_{0} \epsilon}\right) \int_{0}^{n}\left\langle\left(\mathbf{R}_{0 l} \cdot \mathbf{e}_{y}\right)\left(\mathbf{F}_{l} \cdot \mathbf{e}_{x}\right)\right\rangle_{A v} d l} \\
=\left(\zeta N / 1200 M_{0} \eta_{0} \lambda^{2}\right) F(\lambda n), \\
\left\langle\left(\mathbf{R}_{0 k} \cdot \mathbf{e}_{y}\right)\left(\mathbf{F}_{l} \cdot \mathbf{e}_{x}\right)\right\rangle_{A v}=(-\zeta \epsilon / 6)\left\langle\mathbf{R}_{0 k} \cdot \mathbf{R}_{0 l}\right\rangle_{A v} \\
-\left(\zeta / 6 \pi \eta_{0}\right) \int_{0}^{n}\left\langle\left(\mathbf{R}_{0 k} \cdot \mathbf{e}_{y}\right)\left(\mathbf{F}_{s} \cdot \mathbf{e}_{x}\right)_{A v}\left\langle 1 / R_{l s}\right\rangle_{A v} d s,\right.
\end{array}
$$

where $[\eta]$ is the intrinsic viscosity; $\eta_{0}$ is the solvent viscosity; $\zeta$ is the friction constant per unit length; $\epsilon$ is the shear gradient of flow; $M_{0}$ is the molecular weight per unit length; $\mathbf{R}_{0 k}$ and $\mathbf{R}_{0 l}$ are vectors from the mass to positions $k$ and $l$ units distance along the chain; $\mathbf{F}_{l}$ is the force exerted on the solvent by an element of the chain at $l ; \mathbf{e}_{x}$ and $\mathbf{e}_{y}$ are unit vectors in the $x$ and $y$ direction.

The $\left\langle\mathbf{R}_{0 k} \cdot \mathbf{R}_{0 l}\right\rangle_{\mathrm{Av}}$ is easy to calculate, but $\left\langle 1 / \boldsymbol{R}_{l s}\right\rangle_{\mathrm{Av}}$, the kernel of the integral equation, is not. In our treatment it is approximated by the formula

$$
\begin{gathered}
\left\langle 1 / R_{l s}\right\rangle_{\mathrm{Av}}=\left[1 /\left\langle R_{l s}{ }^{2}\right\rangle_{\mathrm{Av}}\right]^{\mathrm{t}} G(\lambda|1-s|) \\
\left\langle R_{l s}{ }^{2}\right\rangle_{\mathrm{Av}}=|l-s| / \lambda-\left(1 / 2 \lambda^{2}\right)[1-\exp (-2 \lambda|l-s|)],
\end{gathered}
$$

where $G$ varies from 1.00 to 1.38 as the particle changes from a rod to a coil form.

It is easy to show that if $\lambda$ is allowed to approach zero, Eq. (1a) assumes the form obtained previously for the rodlike molecule, ${ }^{4,5}$ and as $\lambda$ becomes large, the solution is essentially similar to the result obtained for the random coil. ${ }^{2}$

From Eq. (1a), one can recognize that the intrinsic viscosity may be considered as a product of two functions, one of which depends on $\lambda$ the stiffness parameter and the other on the product of the stiffness parameter and chain length. Multiplying by $n^{2}$ in the numerator and denominator, the intrinsic viscosity may also be expressed as a product of $n^{2}$ and a function of the stiffness parameter times chain length.

The Fredholm equation [Eq. (1b)] which applies to the stiff chain model cannot be solved in terms of known functions as could the problems of the works cited in footnotes 2,4 , and 5 . Instead, numerical computations are being used to obtain a solution. A serious difficulty is encountered because of the mathematical singularity in the kernel of the integral equation. 